\section{Eight common misconceptions about HIV vaccines that may impede progress in controlling the AIDS epidemic}

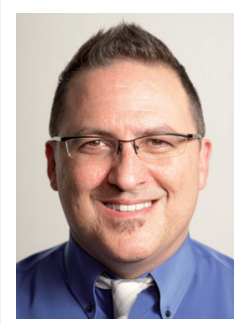

\author{
"The acceptability of future HIV vaccines is not \\ guaranteed; in fact, achieving broad uptake \\ becomes even more consequential in the case of \\ partially efficacious vaccines, which are less \\ acceptable to end users."
}

Peter A Newman

University of Toronto, Factor-Inwentash Faculty of Social Work, 246 Bloor Street West, Toronto, ON M5S 1Y4, Canada = Tel.. +7 4169468671 =p.newman@utoronto.ca
The discovery of a safe and efficacious HIV vaccine would be among the most significant contributions to global health in the early 21 st century. Despite current prevention methods and groundbreaking advances in antiretroviral treatment, an estimated 2.5 million people worldwide are infected with HIV every year [101]. In the USA, annual HIV incident infections (estimated 55,000) have not abated for two decades [1]. In low- and middle-income countries, new infections continue to outpace access to treatment [101], and many persons at risk lack access to existing prevention technologies [2].

New and emerging HIV prevention technologies, including antiretroviral-based oral preexposure prophylaxis and topical microbicides, may yield substantial contributions to controlling the epidemic. Nevertheless, fundamental sociobehavioral and structural challenges, which continue to dilute the effectiveness of existing prevention technologies requiring daily or pericoital usage, suggest that a vaccine is needed to reverse HIV on a population level.

The purpose of identifying HIV vaccine misconceptions is neither to discredit vaccine research nor to devalue the tremendous global importance of a HIV vaccine. Rather, identification of gaps in our conceptions and practices may reveal opportunities to promote the success of the HIV vaccine endeavor.

\section{Misconception 1: efficacy in clinical trials will translate to effectiveness in the real world}

HIV vaccine development faces tremendous scientific challenges. Indeed, as the argument goes, without addressing these we may not have the luxury of dealing with the mundane challenges of implementation. However, to identify clinical trial efficacy as the end game allows predictable and formidable, yet surmountable challenges to hamper or even undermine the success of a vaccine in controlling the epidemic.

"...in the absence of active
communication and ongoing
engagement with information from
bona fide scientific and public
health sources, the knowledge
vacuum is more likely to be filled with
sensationalistic media stories,
conspiracy theories and mistrust."

A quick overview of vaccines that are currently available, particularly adult vaccines for diseases that can be sexually or parenterally transmitted, supports the importance of an implementation science approach [3]. Coverage for highly efficacious hepatitis B vaccines, licensed for over 20 years, is estimated at $10-30 \%$ among people who inject drugs in New York City, NY, USA [4], with wide variability $(3-70 \%)$ reported among men who have sex with men in the USA [5] groups at high risk for HIV infection [1]. HPV vaccine coverage remains suboptimal among US women (48.7\%) and men (1.4\%) aged 13-17 years, amidst ongoing concerns about safety, efficacy, behavioral disinhibition and cost-effectiveness [6]. Vaccine efficacy does not ensure effectiveness. An implementation science approach directs us to fund and advance rigorous scientific inquiry - before a product is publicly available - to address ubiquitous gaps

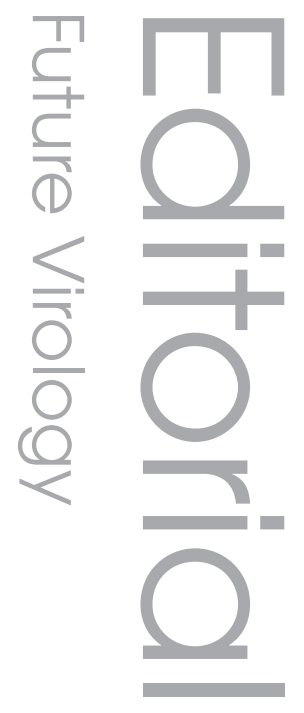

(n)

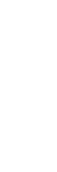


between product efficacy in clinical trials and effectiveness in the real world [3].

\section{Misconception 2: once a licensed HIV vaccine is made accessible, people will line up to be vaccinated}

Although it is vital to address organizational and structural challenges for future HIV vaccine dissemination and implementation at legal, economic, political and institutional levels, vaccine acceptability among end users remains an indelible part of the equation [7]. A systematic review of 20 studies $(n=7576)$ identified HIV vaccine acceptability among potential end users ranging from 37 to 94 on a 100 -point scale, with significantly lower acceptance of a moderate $(50 \%)$ efficacy $(40.4 / 100)$ versus a high (80-95\%) efficacy vaccine $(73.8 / 100)$ [7]. Concerns about vaccine efficacy and safety, perceptions of HIV risk and vaccine fears were significantly associated with acceptability. The acceptability of future HIV vaccines is not guaranteed; in fact, achieving broad uptake becomes even more consequential in the case of partially efficacious vaccines, which are less acceptable to end users.

\section{Misconception 3: we should not disclose too much information about HIV vaccine development \& clinical trials as the general public cannot handle it}

Although this view is certainly not unanimous, concerns that providing piecemeal information to the general public will result in the fueling of misconceptions and false hopes may support the withholding of information. However, in the absence of active communication and ongoing engagement with information from bona fide scientific and public health sources, the knowledge vacuum is more likely to be filled with sensationalistic media stories, conspiracy theories and mistrust $[8,9]$. In addition to counteracting myths and misconceptions that portend danger and malevolence on the part of medical researchers and the government, it is important to temper unrealistic hopes for HIV vaccine effectiveness. Magical expectations may promote an 'early idealization, sudden condemnation' phenomenon, which contributed to delicensure of a previously released rotavirus vaccine [9]. In the case of a partially efficacious HIV vaccine, initial breakthrough infections may not only call into question the value of vaccination; they might be wrongfully attributed to the vaccine itself, which could pose substantial obstacles to uptake [10].

\section{Misconception 4: a HIV vaccine will obviate the complex sociobehavioral challenges that arise in preventing an infectious disease largely spread by sex \& injecting drug use, which disproportionately affects poor \& marginalized populations \& the developing world}

HIV vaccines would mitigate many of the thorny real-world challenges that pose obstacles to the effectiveness of condoms and are likely to dilute the effectiveness of pre-exposure prophylaxis regimens requiring daily usage. Yet, there is no vaccine to inoculate against sociostructural obstacles that arise due to poverty, lack of access to healthcare, healthcare discrimination, HIV stigma, and discrimination against women and sexual minorities. The development of evidence-informed interventions to address what are likely to be pervasive obstacles to HIV vaccine coverage may not only enhance vaccine effectiveness, but may increase the effectiveness of existing HIV prevention regimens, both behavioral and biomedical.

\section{Misconception 5: HIV vaccine trials are a biomedical endeavor}

Despite premature biomedical HIV prevention trial shutdowns in low- and middle-income countries that might have been averted with: more extensive community engagement and preparedness [11,12]; inconsistent adherence to product usage that has posed serious threats to efficacy in clinical trials and interpretation of trial results [13]; potential harms due to risk behavior increases among trial volunteers as a result of preventive misconception [14]; and documented social impacts of trial participation [15], HIV vaccine trial teams are characterized by minimal (if any) inclusion of social scientists. Meaningful integration of social scientists on HIV vaccine trial teams across the trajectory of clinical trials, from community preparedness and protocol development through post-trial dissemination of information, is vital to address the sociobehavioral challenges of clinical trial implementation. Beyond supporting the safe, ethical and successful conduct of clinical trials, funding and integration of social science research in the context of HIV vaccine trials may also promote evidence to support future dissemination of post-licensure HIV vaccines. 


\section{Misconception 6: given high rates of new infections among many populations, a sufficient number of volunteers will enroll in HIV vaccine clinical trials for the foreseeable future}

Despite the best of intentions, it is the nature of clinical trials that they often do not result in efficacious products; sometimes they result in unexpected harms [16]. Studies of trial participants $[17,18]$, most-at-risk populations [19] and civil society representatives [20] conducted in the wake of HIV vaccine trials that were terminated early reveal mixed reactions. Some participants reported sustained trust in trial investigators and HIV vaccine research, and strong willingness to participate in future research; others retroactively doubted the integrity of the informed consent process, felt betrayed and indicated they would not participate in future trials. The evidence suggests that continued willingness to participate cannot be taken for granted. Greater attention to maintaining transparency in the informed consent process, positive personal relationships with trial staff, and open and ongoing two-way communication between investigators and participants during and after trials are key elements of sustaining positive relationships with potential volunteers and communities [17-20].

\section{Misconception 7: introduction of a HIV vaccine will result in risk compensation}

Sociobehavioral [21] and mathematical modeling [22] studies indicate the possible impact of increased post-vaccination risk behaviors in response to the belief that one is protected against HIV infection. Risk compensation is indeed an important phenomenon that could theoretically result in a worsened post-vaccine epidemic scenario. But it is far from a fait accompli. Rigorous investigations of risk compensation in the context of existing and future biomedical prevention technologies is precisely important to support evidence-informed interventions to mitigate this modifiable risk [23].

\section{Misconception 8: HIV vaccine misconceptions are the unique provenance of nonscientists}

An overview of several important, some pervasive, misconceptions toward HIV vaccines, as evidenced in our current practices of funding research, implementing trials and often, hoping for the best, highlights what may be another common misconception: that the most consequential HIV vaccine misconceptions originate from the general public. However, misconceptions among biomedical and clinical researchers, clinicians, policymakers, public health and social service professionals and research funders may enable what might otherwise be modifiable barriers to negatively impact on HIV vaccine trials and the future effectiveness of vaccines in controlling the epidemic.

To the extent we address common misconceptions that constrain scientists and researchers in advancing HIV vaccines, we can thereby unleash the full potential of social and biomedical science to serve the global HIV vaccine endeavor. We may also achieve welcome collateral benefits by helping to counter HIV vaccine misconceptions that might be more easily attributed solely to lack of scientific literacy on the part of the general public.

\section{Financial \& competing interests disclosure}

The author's research is supported by grants from the Canadian Institutes of Health Research/Canadian HIV Vaccine Initiative and the Canada Research Chairs program. The author has no other relevant affiliations or financial involvement with any organization or entity with a financial interest in or financial conflict with the subject matter or materials discussed in the manuscript apart from those disclosed.

No writing assistance was utilized in the production of this manuscript.

\section{References}

1. Hall HI, Song R, Rhodes P et al. Estimation of HIV incidence in the United States. JAMA 300, 520-529 (2008).

2. Gayle HD. Curbing the global AIDS epidemic. N. Engl. J. Med. 348, 1802-1805 (2003).

3. Woolf $\mathrm{SH}$. The meaning of translational research and why it matters. JAMA 299(2), 211-213 (2008)

4. Amesty S, Ompad DC, Galea S et al. Prevalence and correlates of previous hepatitis
$B$ vaccination and infection among young drug-users in New York City. J. Community Health 33(3), 139-148 (2008).

5. Matthews JE, Stephenson R, Sullivan PS. Factors associated with self-reported HBV vaccination among HIV-negative MSM participating in an online sexual health survey: a cross-sectional study. PLoS One 7(2), E30609 (2012).

6. Stupiansky NW, Alexander AB, Zimet GD. Human papillomavirus vaccine and men: what are the obstacles and challenges? Curr. Opin. Infect. Dis. 25(1), 86-91 (2012).
7. Newman PA, Logie C. HIV vaccine acceptability: a systematic review and meta-analysis. AIDS 24(11), 1749-1756 (2010).

8. Roberts KJ, Newman PA, Duan N, Rudy ET. HIV vaccine knowledge and beliefs among communities at elevated risk: conspiracies, questions and confusion. J. Natl Med. Assoc. 97(12), 1662-1671 (2005).

9. Danovaro-Holliday MC, Wood AL, LeBaron $\mathrm{CW}$. Rotavirus vaccine and the news media, 1987-2001. JAMA 287(11), 1455-1462 (2002). 
10. Newman PA, Seiden D, Roberts K, Duan N. A small dose of HIV? HIV vaccine mental models and risk communication. Health Educ. Behav. 36(2), 321-333 (2009).

11. Singh JA, Mills EJ. The abandoned trials of pre-exposure prophylaxis for HIV: what went wrong? PLoS Med. 2(9), E234 (2005).

12. Newman PA. Towards a science of community engagement. Lancet 367(9507), 302 (2006).

13. van der Straten A, van Damme L, Haberer JE, Bangsberg DR. Unraveling the divergent results of PrEP trials for HIV prevention. AIDS 26(7), F13-F19 (2012).

14. Simon AE, Wu AW, Lavori PW, Sugarman J. Preventive misconception: its nature, presence, and ethical implications for research. Am. J. Prev. Med. 32(5), 370-374 (2007).

15. Allen M, Lau CY. Social impact of preventive HIV vaccine clinical trial participation: a model of prevention, assessment and intervention. Soc. Sci. Med. 66(4), 945-951 (2008).
16. Buchbinder SP, Mehrotra DV, Duerr A et al Efficacy assessment of a cell-mediated immunity HIV-1 vaccine (the Step Study): a double-blind, randomised, placebocontrolled, test-of-concept trial. Lancet 372, 1881-1893 (2008).

17. Frew PM, Mulligan MJ, Hou SI, Chan K, del Rio C. Time will tell: community acceptability of HIV research before and after the "Step Study" vaccine discontinuation. J. Clin. Trials 2, 149-156 (2010).

18. Newman PA, Yim S, Daley A et al. "Once bitten, twice shy": participants perspectives in the aftermath of an early HIV vaccine trial termination. Vaccine 29(3), 451-458 (2011).

19. Newman PA, Logie C, James L et al. "Speaking the dialect": understanding public discourse in the aftermath of an HIV vaccine trial shutdown. Am. J. Public Health 101(9), 1749-1758 (2011).

20. Essack Z, Koen J, Slack C, Lindegger G, Newman PA. Civil society perspectives on negative biomedical HIV prevention trial results and implications for future trials.
AIDS Care doi:10.1080/09540121.656566

(2012) (Epub ahead of print).

21. Newman PA, Lee SJ, Rudy E et al. Preventive HIV vaccine acceptability and behavioral risk compensation among a random sample of high-risk adults in Los Angeles (L.A. VOICES). Health Serv. Res. 44(6), 2167-2179 (2009).

22. Blower SM, McLean AR. Prophylactic vaccines, risk behavior change and the probability of eradicating HIV in San Francisco. Science 265, 1451-1454 (1994).

23. Eaton LA, Kalichman SC. Risk compensation in HIV prevention: implications for vaccines, microbicides, and other biomedical HIV prevention technologies. Curr. HIVIAIDS Rep. 4(4), 165-172 (2007).

\section{Website}

101. UNAIDS Report on the Global AIDS Epidemic 2010. www.unaids.org/globalreport/Global_ report.htm 